\title{
Fast transient stability assessment of large power system using probabilistic neural network with feature reduction techniques
}

\begin{abstract}
This paper presents transient stability assessment of a large 87-bus system using a new method called the probabilistic neural network (PNN) with incorporation of feature selection and extraction methods. The investigated power system is divided into smaller areas depending on the coherency of the areas when subjected to disturbances. This is to reduce the amount of data sets collected for the respective areas. Transient stability of the power system is first determined based on the generator relative rotor angles obtained from time domain simulations carried out by considering three phase faults at different loading conditions. The data collected from the time domain simulations are then used as inputs to the PNN. Feature reduction techniques are then incorporated to reduce the number of features to the PNN which is used as a classifier to determine whether the power system is stable or unstable. It can be concluded that the PNN with the incorporation of feature reduction techniques reduces the time taken to train the PNN without affecting the accuracy of the classification results.
\end{abstract}

Keyword: Transient stability assessment; Probabilistic neural network; Correlation analysis; Principle component analysis 